\title{
Vertebroplastia percutânea: uma efetiva técnica cirúrgica minimamente invasiva*
}

\author{
Percutaneous vertebroplasty: an effective, \\ minimally invasive surgical technique
}

Nicolas Gerardo Gómez Cordero', Jesus Ovídio Roberto Gómez Cordero², Luís Alfredo Gómez Vieira³

\section{RESUMO}

Objetivo: Apresentar a nossa experiência no Hospital Português da Bahia com a vertebroplastia percutânea, observando a evolução clínica e radiográfica dos pacientes. Métodos: Foram analisados, retrospectivamente, os dados de 25 pacientes com fraturas por compressão dos corpos vertebrais, cinco homens e 20 mulheres com idade entre 65 e 88 anos de idade, submetidos à vertebroplastia percutânea entre 2003 e 2006, observando-se seus resultados e complicações. Os critérios de inclusão consistiram em: dor intensa na coluna vertebral com impotência funcional conseqüente à fratura recente; falha no tratamento conservador - medicação analgésica e fisioterapia - e pacientes com estabilidade clínica compatível com a submissão de procedimento anestésico. Entre os pacientes, 22 apresentavam fraturas resultantes de osteoporose, um era

* Trabalho realizado no Hospital Português da Bahia - HPBA, Salvador (BA), Brasil.

1. Cirurgião de Coluna do Hospital Português da Bahia - HPBA, Salvador (BA), Brasil.

2. Neurocirurgião do Hospital Português da Bahia - HPBA, Salvador (BA), Brasil.

3. Cirurgião Ortopedista do Hospital Português da Bahia - HPBA, Salvador (BA), Brasil.

Endereço para correspondência: Nicolas Gerardo Gómez Cordero, Av. Anita Garibaldi, 1.133, Ondina - 40210-070 - Salvador, BA. Fax: (71) 3235-2021. E-mail: nicolasgerardoboli@ hotmail.com Recebido em 5/6/07. Aprovado para publicação em 24/1/08. Copyright RBO2008 portador de hemangioma, outro apresentou fratura por linfoma e dois sofreram fratura vertebral por mieloma múltiplo. Os pacientes foram acompanhados semanalmente no primeiro mês e a cada três meses, com um mínimo de 12 meses e um máximo de 36 meses após o procedimento percutâneo. $A$ avaliação quanto à dor, atividade de vida diária $\mathrm{e}$ necessidade do uso de analgésicos foi realizada por um dos autores, baseada em questionário subjetivo para tal, previamente elaborado. Resultados: Foram observados resultados clínicos de excelentes a bons em 23 pacientes $(92 \%)$ e regulares a maus em dois pacientes $(\mathbf{8 \%})$. Houve deterioração por complicação em um caso. Todos os pacientes $(\mathbf{1 0 0 \%})$ retornaram às suas atividades prévias; quatro pacientes $(\mathbf{1 6 \%})$ mudaram a prática das suas atividades de vida diárias. Conclusão: A vertebroplastia percutânea é uma técnica cirúrgica minimamente invasiva que proporcionou, em nossa casuística, rápido e significativo alívio da dor e melhora da qualidade das atividades de vida diária dos pacientes submetidos a esse procedimento.

Descritores - Fratura da coluna vertebral; Osteoporose; Coluna vertebral/cirurgia

\section{ABSTRACT}

Objective: To present the authors' experience at the Portuguese Hospital in Bahia, Brazil, with the percutaneous vertebroplasty, showing the clinical and radiographic evolution of the patients. Methods: Retrospective analysis of the data of 25 patients with 
compression fracture of the vertebrae, five male and 25 female, ages ranging from 65 to 88 years, submitted to percutaneous vertebroplasty between 2003 and 2006. Results and complications were observed. Inclusion criteria were: intense pain in the spinal column with functional inability as a result of a frequent fracture; failure of the conservative treatment - analgesic medication and physiotherapy - and patients with clinical stability compatible with submission to anesthetic procedure. 22 of the patients had osteoporotic fractures, one had hemangioma, another had a fracture due to a lymphoma, and two had vertebral fracture due to multiple myeloma. Patients were followed weekly during the first month, and then at three month intervals for a minimum of 12 months and a maximum of 36 months after the percutaneous procedure. Evaluation of pain, daily life activities, and the need to take analgesic medication was performed by one of the authors, based on a subjective questionnaire previously prepared for that purpose. Results: Excellent to good clinical results were seen in 23 patients (92\%) and regular to poor results were seen in two patients (8\%). Deterioration due to complication was seen in one case. All patients (100\%) returned to their prior activities; four patients $(16 \%)$ changed the practice of their daily life activities. Conclusion: Percutaneous vertebroplasty is a minimally invasive surgical technique that in our series had quick and meaningful pain relief and improvement in the quality of daily life activities of the patients submitted to this procedure.

Keywords - Spine fracture; Osteoporosis; Spinal column/ surgery

\section{INTRODUÇÃO}

A história da vertebroplastia teve seu início na França em 1984, quando o primeiro procedimento foi realizado, sendo posteriormente publicado em $1987 \mathrm{em}$ periódico de língua francesa ${ }^{(1)}$. No entanto, aquela publicação não chamou grande atenção da comunidade científica internacional. Somente quando uma revisão retrospectiva de 47 vertebroplastias em 29 pacientes com fraturas vertebrais osteoporóticas foi publicada em periódico de língua inglesa, alguns anos mais tarde, maior interesse da comunidade internacional ocorreu. Naquele estudo, $90 \%$ dos pacientes apresentaram significativo alívio imediato da dor após a vertebroplastia $^{(2)}$.

A vertebroplastia percutânea consiste na injeção de cimento ósseo (polimetilmetacrilato) no corpo vertebral comprimido(3) ${ }^{(3)}$ A injeção do cimento ósseo é feita guiada por radioscopia, buscando alívio da dor e estabilização do corpo vertebral fraturado. Quando realizada por profissional bem treinado com observação radioscópica direta, trata-se de uma técnica rápida e segura. Essa técnica estabiliza o corpo vertebral com sucesso, em relação ao alívio da dor, em $75 \%$ a $85 \%$ dos pacientes ${ }^{(4-7)}$.

As fraturas compressivas do corpo vertebral podem estar vinculadas a várias condições patológicas, tais como: hemangioma, mieloma múltiplo, metástase osteolítica e osteoporose primária ou secundária ${ }^{(8)}$. Tais fraturas por compressão são comuns, especialmente nos idosos. São geralmente causadas por osteoporose de moderada a grave. O colapso vertebral osteoporótico provoca dor localizada na coluna vertebral associada com instabilidade e/ou cifose, levando à inabilidade para atividades de vida diária e conseqüente alteração psicossocial.

A osteoporose é uma condição patológica caracterizada pela diminuição da massa óssea e deterioração microarquitetural do tecido ósseo, com conseqüente aumento da fragilidade óssea e suscetibilidade a fratura $^{(9)}$. Histologicamente, com o aumento da idade, a estabilidade do colágeno trabecular diminui e os componentes proteoglicanos do osso apresentam orientação molecular alterada ${ }^{(10-11)}$. A disparidade histológica entre o osso normal e o osso osteoporótico é o resultado das diferenças na função celular ${ }^{(12)}$.

A principal manifestação da osteoporose é a fratura patológica. Pacientes osteoporóticos desenvolvem fraturas quando força mínima é aplicada ao osso descontínuo e enfraquecido ${ }^{(13)}$. Embora as fraturas por compressão aguda do corpo vertebral sejam dolorosas, a intensidade da dor é variável. O risco de desenvolvimento da dor crônica aumenta com o número de fraturas vertebrais ${ }^{(14)}$. 
A cifose aumentada e as fraturas por compressão vertebral reduzem a força do tronco ${ }^{(15)}$. Inatividade e fraqueza aumentadas aceleram a perda da massa óssea, acrescentando risco adicional de fraturas ${ }^{(16-17)}$.

\section{MÉTODOS}

Foram analisados, retrospectivamente, os dados de 25 pacientes com fraturas por compressão dos corpos vertebrais, cinco homens e 20 mulheres com idade entre 65 e 88 anos de idade, submetidos à vertebroplastia percutânea entre 2003 e 2006, observando-se seus resultados e complicações, no Hospital Português da Bahia, após aprovação pelo Comitê de Ética da instituição. Os critérios de inclusão consistiram em: dor intensa na coluna vertebral com impotência funcional conseqüente de fratura recente; falha no tratamento conservador - medicação analgésica e fisioterapia - e pacientes com estabilidade clínica para submissão a procedimento anestésico. Os critérios de exclusão consistiram em: fraturas instáveis e presença de: infecção, distúrbio neurológico, compressão medular ou de raiz nervosa, alergia ao meio de contraste iodado, coagulopatia e gravidez.

Metodologicamente, os pacientes foram submetidos a um questionário de avaliação que levava em consideração três itens: a) dor - intensa, moderada ou leve/ mínima; b) capacidade ou não para exercer atividades de vida diária: vestir-se, locomover-se, alimentar-se, asseio pessoal; c) uso de medicação analgésica - constante, intermitente ou desnecessário. Apenas os pacientes com dor intensa ou moderada, incapazes para as atividades de vida diárias e em uso constante de analgésicos, foram submetidos a este estudo.

Os pacientes foram acompanhados semanalmente no primeiro mês e a cada três meses, com um mínimo de 12 meses e um máximo de 36 meses após o procedimento percutâneo. Entre os pacientes, 22 apresentavam fraturas resultantes de osteoporose (figura 1), um paciente era portador de hemangioma (fratura em L3), outro apresentou fratura por linfoma (fratura em T8) e dois sofriam de fraturas vertebrais por mieloma múltiplo (fraturas em T8, T11, L1, L3 e L5). Dos 22 pacientes com fratura por osteoporose, oito apresentavam fratura da vértebra L1, cinco na vértebra L3, dois na

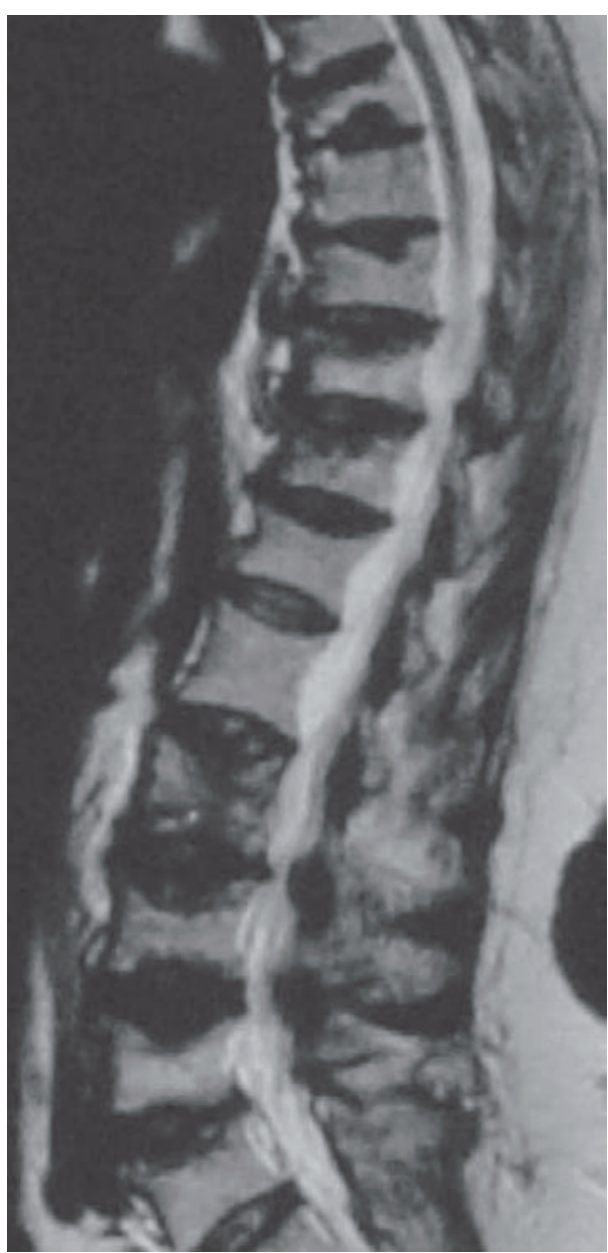

Figura 1 - RM: Corte sagital mostrando fratura vertebral por osteoporose.

vértebra L5, quatro na vértebra T8 e três na vértebra T11.

Em três pacientes, a vertebroplastia foi realizada em múltiplos níveis. Um paciente apresentava fratura por osteoporose em T8, T11, L3 e L5. Os outros dois pacientes, com mieloma múltiplo, apresentavam fratura dos corpos vertebrais: o primeiro, em T11, L1, L3 e L5 e o outro, em T8, T11, L1, L3 e L5.

Quando foi necessário o procedimento em mais de três níveis, realizamos a cirurgia em dois tempos, com intervalo de uma semana entre cada um dos procedimentos.

A técnica cirúrgica da vertebroplastia empregada foi similar àquelas utilizadas para discografia ou excisão discal percutânea por via artroscópica.

Em ambas as regiões, torácica e lombar, a abordagem extrapedicular foi aplicada para ter acesso ao corpo 


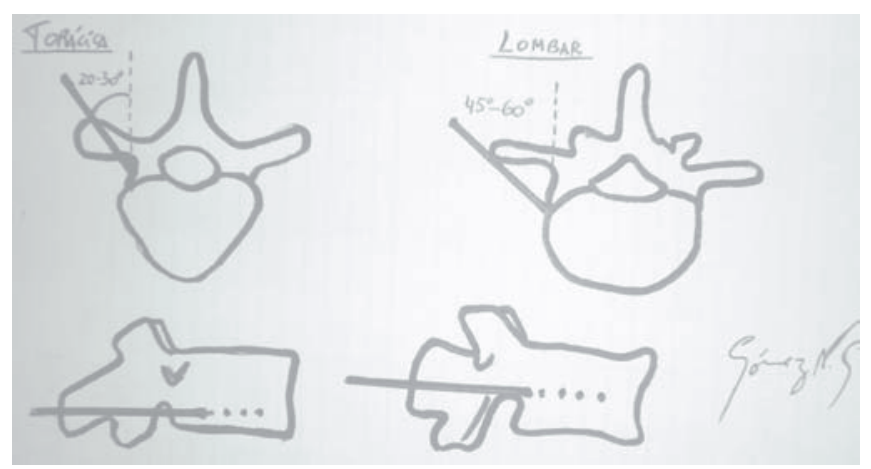

Figura 2 - Desenho esquemático dos ângulos de introdução do fio-guia torácico e lombar

vertebral através de uma incisão percutânea simples. No entanto, os ângulos para introdução dos fios-guia no corpo vertebral são um pouco diferentes nas duas regiões. Os ângulos de acesso à coluna torácica são diferentes dos ângulos de acesso à lombar; eles são mais verticais e os pontos de entrada são mais mediais para evitar complicações como o pneumotórax. Cuidado especial deve ser tomado para evitar a penetração do fio-guia na cortical oposta do corpo vertebral, atingindo as estruturas adjacentes.

Previamente à vertebroplastia, avaliamos o caso clínico por meio de raios $\mathrm{X}$ simples (incidências ânteroposterior e perfil), tomografia computadorizada, ressonância magnética (RM) e cintilografia para determinar a lesão óssea, o grau de cifose ou lordose e a presença de qualquer deformidade como a escoliose.

A posição do paciente para a realização da vertebroplastia varia de decúbito ventral a lateral sobre mesa radiolucente para facilitar a radioscopia. Nossa preferência é pelo decúbito ventral, colocando dois coxins axilares e um coxim longo de apoio ilíaco, o que, por gravidade, facilita a "redução" da fratura.

Utilizamos, para esse procedimento, o kit de vertebroplastia Vertebroplastic ${ }^{\circledR}$ (Johnson-Johnson) cujo cimento ortopédico apresenta reação térmica bem inferior à dos cimentos ortopédicos comuns, o que nos oferece maior segurança ao reduzir o risco de complicações por lesão térmica.

Para a vertebroplastia lombar, identificamos sob radioscopia a linha média da coluna com um fio-guia; tomando por referência esse fio, era traçada uma linha

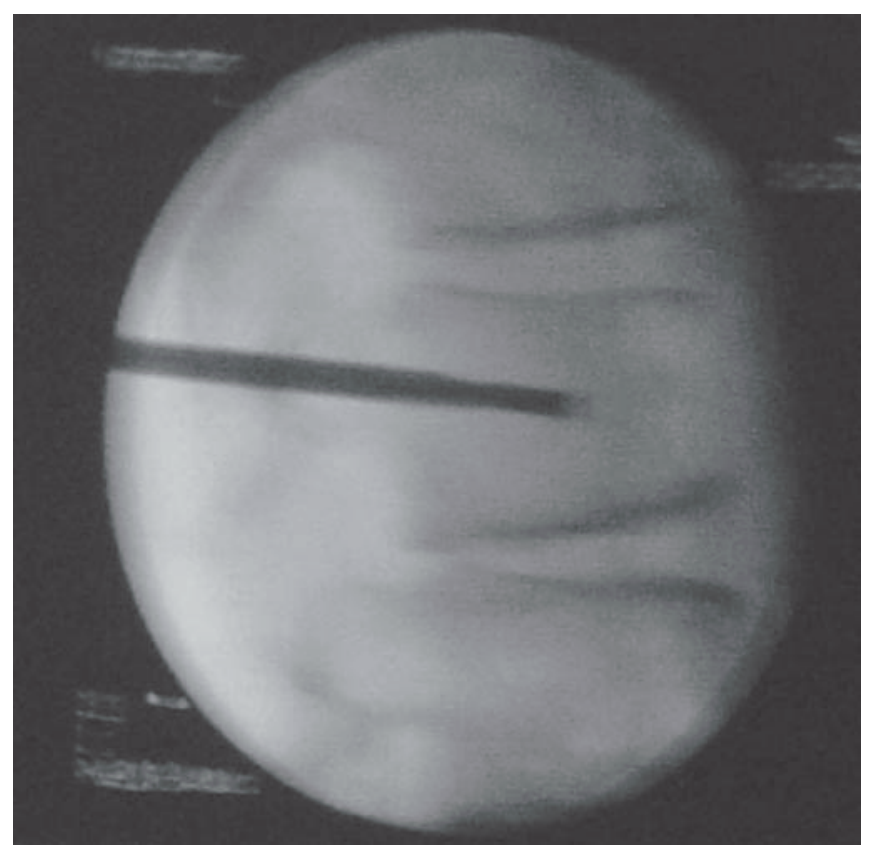

Figura 3 - Penetração da cânula na vértebra sob radioscopia

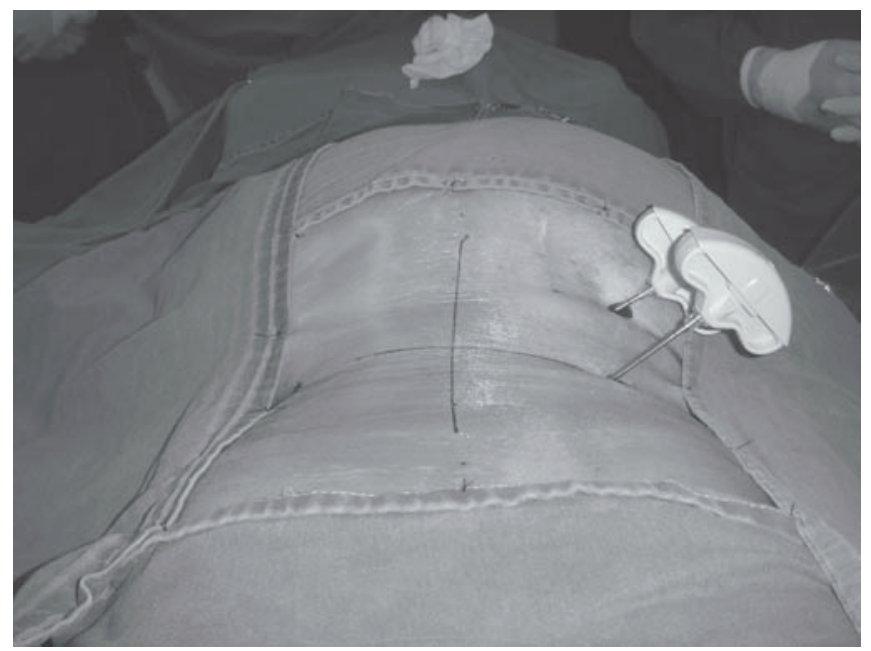

Figura 4 - Visão externa das cânulas posicionadas

na pele com caneta marcadora. A seguir, é riscada outra linha transversa bissecando a apófise transversa e o pedículo. Após medir a distância de 8 a $10 \mathrm{~cm}$ laterais à linha média inicial, é desenhada uma nova linha paralela àquela. Um fio-guia sob visão radioscópica ântero-posterior é introduzido em um ângulo entre $45^{\circ}$ e $60^{\circ}$ a partir do eixo vertical da linha média inicial $\left(\right.$ L1 a L3 $=>45^{\circ}-50^{\circ} ;$ L4 $=>50^{\circ}-55^{\circ} ;$ L5 $\left.=>55^{\circ}-60^{\circ}\right)$ 


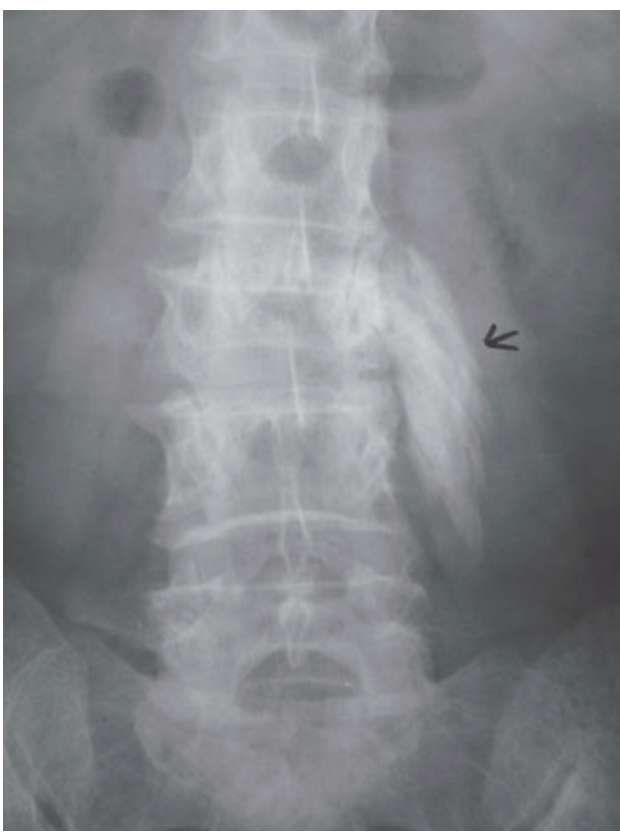

Figura 5 - Extravasamento do cimento ortopédico (seta)
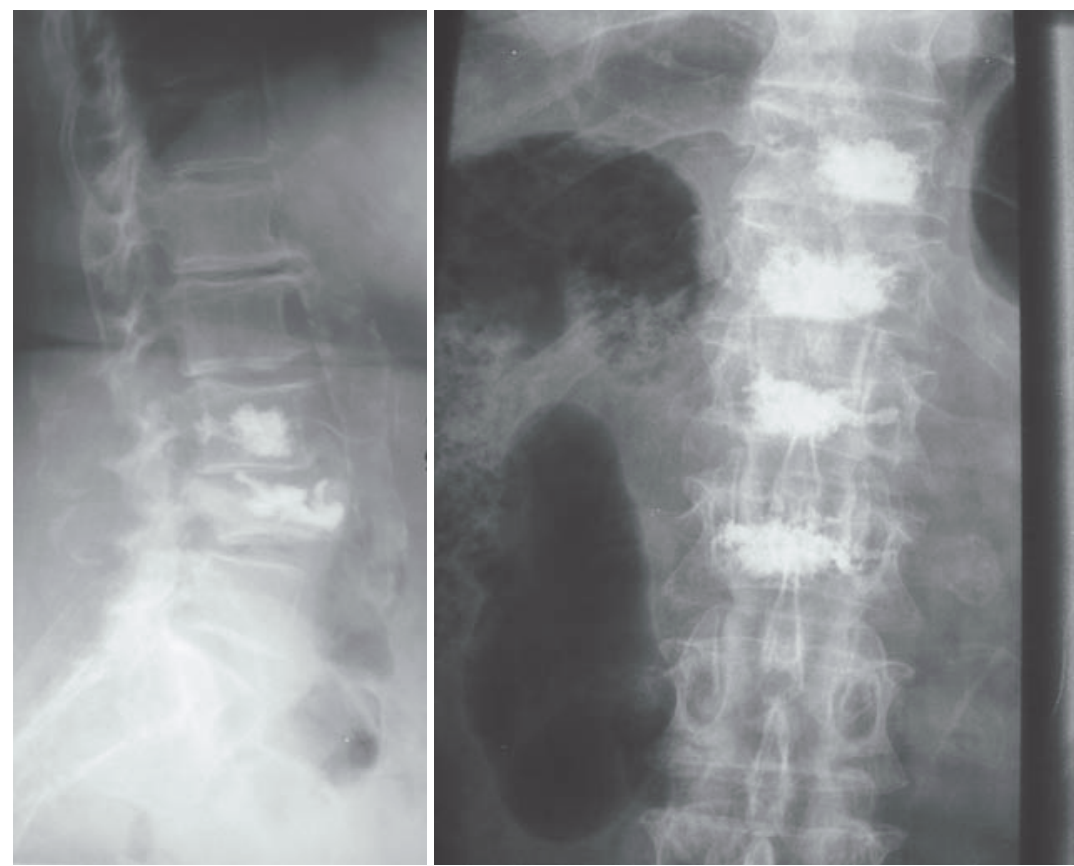

Figuras 6 e 7 - Vertebroplastia lombar e torácica em diferentes níveis (figura 2). Estando bem posicionado, esse fio-guia era avançado até alcançar 70\% do corpo vertebral sob visão lateral (perfil).

Tendo esse fio como guia, a cânula de aplicação de cimento ósseo é posicionada no interior do corpo vertebral comprometido (figuras 3 e 4). Para evitar o eventual extravasamento do cimento (figura 5), injetamos material de contraste radiolucente líquido pela cânula. Tal medida nos mostra se a posição da cânula está correta ou se corremos o risco de injetar o cimento ósseo no interior do canal medular por extravasamento, o que pode levar a sérias complicações. Em seguida, são injetados de 2 a $5 \mathrm{ml}$ de cimento ortopédico.

A técnica cirúrgica para a vertebroplastia torácica foi semelhante à técnica da vertebroplastia lombar, diferindo apenas na distância da linha paralela à linha vertical, que é de 2 a $2,5 \mathrm{~cm}$ lateralmente, e no ângulo de introdução do fio-guia na vértebra, que é entre $20^{\circ}$ e $30^{\circ}$.

Importante ressaltar que esta técnica apresenta algumas contra-indicações, como infecção, gravidez, coagulopatias, vértebra plana, fraturas instáveis, distúrbios neurológicos, compressão medular ou de raiz nervosa.
A análise estatística deste trabalho foi baseada na metodologia do teste $t$ de Student, obtendo os dados pré e pós-operatoriamente. Os resultados foram considerados significativos se $\mathrm{p}<0,05$.

\section{RESULTADOS}

O tempo de seguimento para avaliação desses pacientes foi de 20 e 36 meses (média de 28 meses). Em relação ao tempo médio da nossa observação, consideramos o resultado excelente quando os pacientes, após o procedimento, voltaram ao seu estado funcional prévio à lesão sem qualquer sensação de dor $(83 \%$ dos casos). O resultado foi considerado como bom nos pacientes que voltaram ao estado funcional prévio à lesão, porém, com alguma sensação de dor residual (9\% dos casos). E considerado regular (4\%), quando os pacientes apresentaram limitação funcional e dor residual, no entanto, de menor intensidade que o observado na sua avaliação pré-operatória. $\mathrm{O}$ resultado foi considerado como mau quando permaneceram com limitação funcional importante e não houve melhora da dor (4\% dos casos).

Foram observados resultados clínicos excelentes e bons em 23 pacientes (92\%); e regular e mau em dois 
(8\%). Houve deterioração transitória, por complicação, em um caso. Todos os pacientes $(100 \%)$ retornaram às suas atividades prévias; quatro $(16 \%)$ mudaram a prática das suas atividades de vida diárias, porém, plenamente satisfeitos com o resultado pós-operatório.

Estatisticamente, observou-se na avaliação pré-operatória um escore de 75,1 e após três meses das cirurgias este escore diminuiu para 38,7 ( $\mathrm{p}<0,0001)$. Toda análise estatística foi realizada usando o SPSS Statistical Software (versão 9.0, SPSS Inc., Chicago, IL ${ }^{\circledR}$ ).

Um paciente apresentou complicação por extravasamento do cimento para o interior do canal medular em grande quantidade, o que nos obrigou a proceder à cirurgia aberta ampla de imediato para retirada do mesmo. A paciente evoluiu com pé eqüino paralítico, paralisia que regrediu totalmente após seis meses.

Dos 25 pacientes submetidos à vertebroplastia percutânea, dois faleceram em decorrência de complicações relacionadas com suas doenças de base (linfoma e mieloma múltiplo). No entanto, todos os indivíduos, quando consultados quanto à necessidade de repetição do procedimento, se necessário, foram unânimes em consentir um novo ato cirúrgico.

\section{DISCUSSÃO}

As fraturas vertebrais por osteoporose podem passar despercebidas, com sintomas mínimos ou podem levar a consequiências físicas, psicológicas e sociais que podem profundamente afetar a saúde relacionada à qualidade de vida. Na maioria dos casos, os procedimentos convencionais para essas fraturas resolvem o problema entre três e oito semanas. Entretanto, tais fraturas levam alguns pacientes à diminuição das atividades físicas, imobilidade, isolamento social e depressão em alguns ${ }^{(18-20)}$.

A vertebroplastia é técnica que oferece alternativa para o tratamento das fraturas vertebrais. Vários estudos têm demonstrado os benefícios desse procedimento, com bons resultados ${ }^{(2-3,7,21-23)}$.

No nosso estudo, todos os 25 pacientes foram submetidos à vertebroplastia após período mínimo de dois a seis meses sem regressão dos sintomas com o tratamento conservador, com resultados pós-operatórios bastante animadores.
Autores têm postulado que a vertebroplastia pode aumentar a incidência de novas fraturas nas vértebras $\operatorname{adjacentes}^{(24-25)}$. Não observamos tal problema neste estudo, talvez devido ao curto tempo de acompanhamento dos nossos pacientes.

As técnicas atuais não impedem consistentemente o extravasamento do cimento para locais indesejáveis. Cotten et al mostraram a ocorrência dessa complicação em 29 de 40 pacientes reavaliados radiologicamente no pós-operatório, mas apenas dois necessitaram de nova intervenção ${ }^{(26)}$.

Observamos extravasamento do cimento em dois pacientes. Em um dos casos foi necessária reintervenção cirúrgica aberta de imediato devido ao extravasamento do cimento ortopédico para o interior do canal medular. Esse extravasamento levou a um comprometimento neurológico distal que, felizmente, regrediu, normalizando-se após seis meses do procedimento cirúrgico. Com o objetivo de evitar novos extravasamentos, passamos a injetar contraste líquido radiolucente, buscando observar a penetração deste no corpo vertebral e qualquer eventual extravasamento para locais indesejáveis. Assim, ao constatarmos tal extravasamento, passamos a corrigir o posicionamento da cânula/ trocarte no corpo vertebral antes de injetarmos o cimento ósseo. A partir de então, não mais observamos extravasamento do cimento nos nossos casos subseqüentes.

Além do extravasamento, outras complicações podem ocorrer com a técnica de vertebroplastia, como óbito, sangramento importante, lesão vascular, infecção, pneumotórax, hemotórax, lesão neurológica, embolia pulmonar $^{(27)}$.

O cimento ósseo ideal deve ser biodegradável, não tóxico, de baixa temperatura e com reação biomecânica o mais próximo do osso humano ${ }^{(28)}$. Essas as características do cimento por nós utilizado. Além disso, é importante observar a consistência desse cimento quando da injeção no corpo vertebral. Quanto mais líquido for o cimento, maior o risco de extravasamento. Portanto, o cimento ósseo deve ser introduzido o mais pastoso possível, tipo "pasta de dente".

O objetivo deste artigo foi destacar a técnica da vertebroplastia como um real instrumento de tratamento 
das fraturas por compressão do corpo vertebral. Outros estudos sobre a vertebroplastia também ratificam a utilidade deste procedimento cirúrgico ${ }^{(21,29-30)}$.

Este procedimento proporciona o maior benefício aos pacientes com fratura vertebral por compressão quando comparados com os tratados conservadoramente.

\section{CONCLUSÃO}

A vertebroplastia percutânea é uma técnica cirúrgica minimamente invasiva que demonstrou rápido e significativo alívio da dor e melhora da qualidade das atividades de vida diária dos pacientes submetidos a este procedimento. Quando realizada por profissional bem treinado com observação radioscópica direta, é uma técnica rápida e segura.

\section{REFERÊNCIAS}

1. Galibert P, Deramond H, Rosat P, le Gars D. Preliminary note on the treatment of vertebral angioma by percutaneous acrylic vertebroplasty. Neurochirurgie. 1987;33(2):166-8.

2. Jensen ME, Evans AJ, Mathis JM, Kallmes DF, Cloft HJ, Drio JE. Percutaneous polymethylmethacrylate vertebroplasty in the treatment of osteoporotic vertebral body compression fractures: technical aspects. AJNR Am J Neuroradiol. 1997; 18(10):1897-904

3. Deramond H, Depriester C, Galibert P, Le Gars D. Percutaneous vertebroplasty with polymethylmethacrylate: technique, indications, and results. Radiol Clin North Am. 1998;36(3):533-46.

4. Mathis JM, Barr JD, Belkoff SM, Barr MS, Jensen ME, Deramond H. Percutaneous vertebroplasty: a developing standard of care for vertebral compression fractures. AJNR Am J Neuroradiol. 2001;22(2):373-81.

5. Mathis JM, Petri M, Naff N. Percutaneous vertebroplasty treatment of steroid-induced osteoporotic compression fractures. Arthritis Rheum. 1998;41(1):171-5.

6. Evans AJ, Jensen ME, Kip KE, DeNardo AJ, Lawler GJ, Negin GA, et al. Vertebral compression fractures: pain reduction and improvement in functional mobility after percutaneous polymethylmethacrylate vertebroplasty retrospective report of 245 cases. Radiology. 2003;226(2):366-72.

7. Barr JD, Barr MS, Lemley TJ, McCann RM. Percutaneous vertebroplasty for pain relief and spinal stabilization. Spine. 2000;25(8):923-8.

8. Lieberman IH, Togawa D, Kayanja MM. Vertebroplasty and kyphoplasty: filler materials. Spine J. 2005;5(6 Suppl):305S$16 \mathrm{~S}$.
9. [No authors listed]. Consensus development conference: diagnosis, prophylaxis, and treatment of osteoporosis. Am J Med. 1993;94(6):646-50.

10. Schnitzer CM. Histomorphology of osteoporosis. In: An YE, editor. Orthopaedic issues in osteoporosis. Boca Raton: CRC; 2003. p. 19-37.

11. Ferris BD, Klenerman L, Dodds RA, Bitensky L, Chayen J. Altered organization of non-collagenous bone matrix in osteoporosis. Bone. 1987;8(5):285-8.

12. Kim CW, Minocha J, Wahl CE, Garfin SR. Response of fractured osteoporotic bone to polymethylacrylate after vertebroplasty: case report. Spine J. 2004;4(6):709-12.

13. Truumees E, Hilibrand A, Vaccaro AR. Percutaneous vertebral augmentation. Spine J. 2004;4(2):218-29.

14. Oleksik A, Lips P, Dawson A, Minshall ME, Shen W, Cooper C, et al. Health related quality of life in postmenopausal women with low bone mineral density with or without prevalent vertebral fractures. J Bone Miner Res. 2000;15(7):1384-92.

15. Sinaki M, Wollan PC, Scott RW, Gelczer RK. Can strong back extensors prevent vertebral fractures in women with osteoporosis? Mayo Clinic Proc. 1996;71(10):951-6.

16. Silverman SL. The clinical consequences of vertebral compression fractures. Bone. 1992;13(Suppl 2):S27-S31.

17. Lindsay R, Silverman SL, Cooper C, Hanley DA, Barton I, Broy SB, et al. Risk of new vertebral fracture in the year following a fracture. JAMA. 2001;285(3):320-3.

18. Lyles KW, Gold DT, Shipp KM, Pieper CF, Martinez S, Mulhausen PL. Association of osteoporotic vertebral compression fractures with impaired functional status. Am J Med. 1993;94(6):595-601.

19. Gold DT. The clinical impact of vertebral fractures: quality of life in women with osteoporosis. Bone. 1996;18(3 Suppl): 185S-9S.

20. Nevitt MC, Thompson DE, Black DM, Rubin SR, Ensrud K, Yates AJ, et al. Effect of alendronate on limited-activity days and bed-disability days caused by back pain in postmenopausal women with existing vertebral fractures. Fracture Intervention Trial Research Group. Arch Intern Med. 2000;160(1):77-85.

21. Cortet B, Cotten A, Boutry N, Flipo RM, Duquesnoy B, Chastanet $\mathrm{P}$, et al. Percutaneous vertebroplasty in the treatment of osteoporotic vertebral compression fractures: an open prospective study. J Rheumatol. 1999;26(10):2222-8.

22. Heini PF, Walchli B, Berlemann U. Percutaneous transpedicular vertebroplasty with PMMA: operative technique and early results. A prospective study for the treatment of osteoporotic compression fractures. Eur Spine J. 2000;9(5): 445-50.

23. Zoarski GH, Snow P, Olan WJ, Stallmeyer MJ, Dick BW, Hebel JR, et al. Percutaneous vertebroplasty for osteoporotic compression fractures: quantitative prospective evaluation of long-term outcomes. J Vasc Interv Radiol. 2002;13(2 Pt 1): $139-48$. 
24. Grados F, Depriester C, Cayrolle G, Hardy N, Deramond H, Fardellone P. Long-term observations of vertebral osteoporotic fractures treated by percutaneous vertebroplasty. Rheumatology (Oxford). 2000;39(12):1410-4.

25. Uppin AA, Hirsch JA, Centenera LV, Pfiefer BA, Pazianos AG, Choi IS. Occurrence of new vertebral body fracture alter percutaneous vertebroplasty in patients with osteoporosis. Radiology. 2003;226(1):119-24.

26. Cotten A, Dewatre F, Cortet B, Assaker R, Leblond D, Duquesnoy B, et al. Percutaneous vertebroplasty for osteolytic metastases and myeloma: effects of the percentage of lesion filling and the leakage of methyl methacrylate at clinical follow-up. Radiology. 1996;200(2):525-30.
27. Álvarez L, Alcaraz M, Pérez-Higueras A, Granizo JJ, De Miguel I, Rossi RE, et al. Percutaneous vertebroplasty. Functional improvement in patients with osteoporotic compression fractures. Spine. 2006;31(10):1113-8.

28. Berlemann U, Ferguson SJ, Nolte LP, Heini PF. Adjacent vertebral failure after vertebroplasty. A biomechanical investigation. J Bone Joint Surg Br. 2002;84(5):748-52.

29. Cyteval C, Sarrabere MP, Roux JO, Thomas E, Jorgensen C, Blotman F, et al. Acute osteoporotic vertebral collapse: open study on percutaneous injection of acrylic surgical cement in 20 patients. AJR Am J Roentgenol. 1999;173(6):1685-90.

30. Martin JB, Jean B, Sugiu K, San Millán Ruiz D, Piotin M, Murphy K, et al. Vertebroplasty: clinical experience and follow-up results. Bone. 1999;25(2 Suppl):11S-5S.

Declaração de inexistência de conflitos de interesse: Declaramos para os devidos fins que não há qualquer conflito de interesse que impeça a publicação deste artigo. 